\title{
Pattern Formation of Hollow Drops from Final Breakup of a Hollow Jet*
}

\author{
Truong V. VU**, Hideyuki TAKAKURA**, John C. WELLS** \\ and Takashi MINEMOTO*** \\ ${ }^{* *}$ Graduate School of Science and Engineering, Ritsumeikan University \\ 1-1-1 Nojihigashi, Kusatsu, Shiga 525-8577, Japan \\ E-mail: gr042084@ed.ritsumei.ac.jp \\ ***Ritsumeikan Global Innovation Research Organization, Ritsumeikan University \\ 1-1-1 Nojihigashi, Kusatsu, Shiga 525-8577, Japan
}

\begin{abstract}
An experimental investigation on the formation and breakup of a hollow jet issuing from a coaxial nozzle into ambient air has been carried out. The hollow jet consists of an outer jet of water that encloses an inner jet of argon gas. By varying the flow rate ratio of the inner jet to the outer jet, three different breakup patterns of hollow water jets are identified using two types of nozzles: (I) mixed hollow and simple drop formation, (II) single-core hollow drop formation, and (III) multi-core hollow drop formation. These patterns are mapped in a space of Weber number versus flow rate ratio, $W e-Q$. Experimental results in pattern (II) show that increasing the flow rate ratio results in increasing the formation frequency, slightly increasing outer diameters of the hollow drops, and decreasing their wall thickness.
\end{abstract}

Key words: Hollow Jet, Hollow Drop, Coaxial Nozzle, Breakup Pattern

\section{Introduction}

Compound liquid jets are found in a large variety of application areas such as encapsulations ${ }^{(1,2)}$, ink-jet printing ${ }^{(3,4)}$, and fiber spinning ${ }^{(5)}$. In these applications, the compound jets consisting of a core fluid surrounded by a coaxial layer of a shell liquid are generally formed by forcing fluids through a coaxial nozzle. Other approaches to form compound jets and drops are based on the use of focusing devices ${ }^{(6,7)}$, and electric forces ${ }^{(8,9)}$ for micro/nano encapsulations and nanofibers. A hollow jet is one type of the compound jet as the core fluid is gas. Such compound (hollow) jets are inherently unstable and eventually decompose into compound (hollow) drops due to the Reyleigh instability ${ }^{(10)}$. The spatial and temporal instability of a viscous compound jet was studied by Chauhan et al. ${ }^{(11)}$ in which the compound jet was initially stationary, and perturbed by an axisymmetric disturbance. The instability and breakup of a moving viscous compound jet was investigated by Shen and $\mathrm{Li}^{(12)}$, and Chen and $\mathrm{Lin}^{(13)}$. Numerical calculations can be found elsewhere ${ }^{(14-16)}$.

Along with the theoretical and numerical studies, there are a great number of experimental investigations on compound liquid jets. Hertz and Hermanrud ${ }^{(4)}$ studied experimentally the dynamics of compound jets, and demonstrated the production of compound drops from breakup of the compound jet. Utada et al. ${ }^{(17)}$ used microfluidic devices to generate double emulsions that contained a single inner drop or many inner drops from coaxial jets. They found that generating a double emulsion with a single inner drop requires both coaxial jets to be simultaneously dripping or simultaneously jetting. Chiu and

[DOl: 10.1299/jfst. 6 .823]

Copyright $(2011$ by JSME 
Lin ${ }^{(18)}$ classified the final breakup patterns of their compound jets of water-in-diesel-in-air into six groups based upon the wave length and the mass ratio. The jets were under periodic excitation. However, it is difficult to observe the formation and detachment of the inner drops in their experiments.

In contrast to the large number of experiments on compound liquid jets, only limited studies focused on hollow jet breakup patterns. Kendall ${ }^{(19)}$ studied how a hollow jet of water surrounding a flow of gas at its core decomposes into hollow drops of uniform thickness in ambient air. Most of the reported results were obtained using one coaxial nozzle. He further studied the effects of the flow rate ratio of the core gas to the shell fluid, and of hollow jet fluid properties on the hollow drop formation, as well as oscillations of the resulting drops. He reported that his case is profoundly different from those in the Rayleigh instability ${ }^{(10)}$ in the amplification rate of the axisymmetric deformation and the uniformity in size of drops. We, however, find that, as discussed later, in some cases satellite drops are also formed from hollow jets. Li and Shen ${ }^{(20)}$ experimentally found three flow regimes for the breakup process of an annular water jet exposed to an inner air stream: bubble (hollow drop) formation, annular jet formation, and atomization. Within the hollow drop formation, they showed that both the jet breakup length and the wavelength decreased as the air-to-water velocity ratio increased. However, we find a different result related to the breakup length, as discussed later.

Motivated by scientific interest in the fluid dynamics of a hollow jet, its encapsulation applications ${ }^{(1,2)}$, and a lack of detailed studies of the hollow jet breakup pattern, in the present study, we use a technique similar to that described in Ref. (2) to identify various final breakup patterns of a hollow jet. The final breakup patterns play an important role because they affect the production yield. We form hollow jets that consist of an outer jet of water surrounding an inner (core) jet of argon gas, using two types of coaxial nozzles, as shown in Fig. 1 (a). We pay attention to the regime of bubble formation ${ }^{(20)}$, and investigate how the formation frequency, breakup length and drop size are affected by the gas-to-water flow rate ratio. We do experiments in air at room temperature (about $22-25{ }^{0} \mathrm{C}$ ).

Nomenclature

$\begin{array}{lll}d & : & \text { diameter, } \mathrm{m} \\ D & : & \text { diameter ratio of the coaxial nozzle } \\ f & : & \text { formation frequency, } \mathrm{Hz}\end{array}$
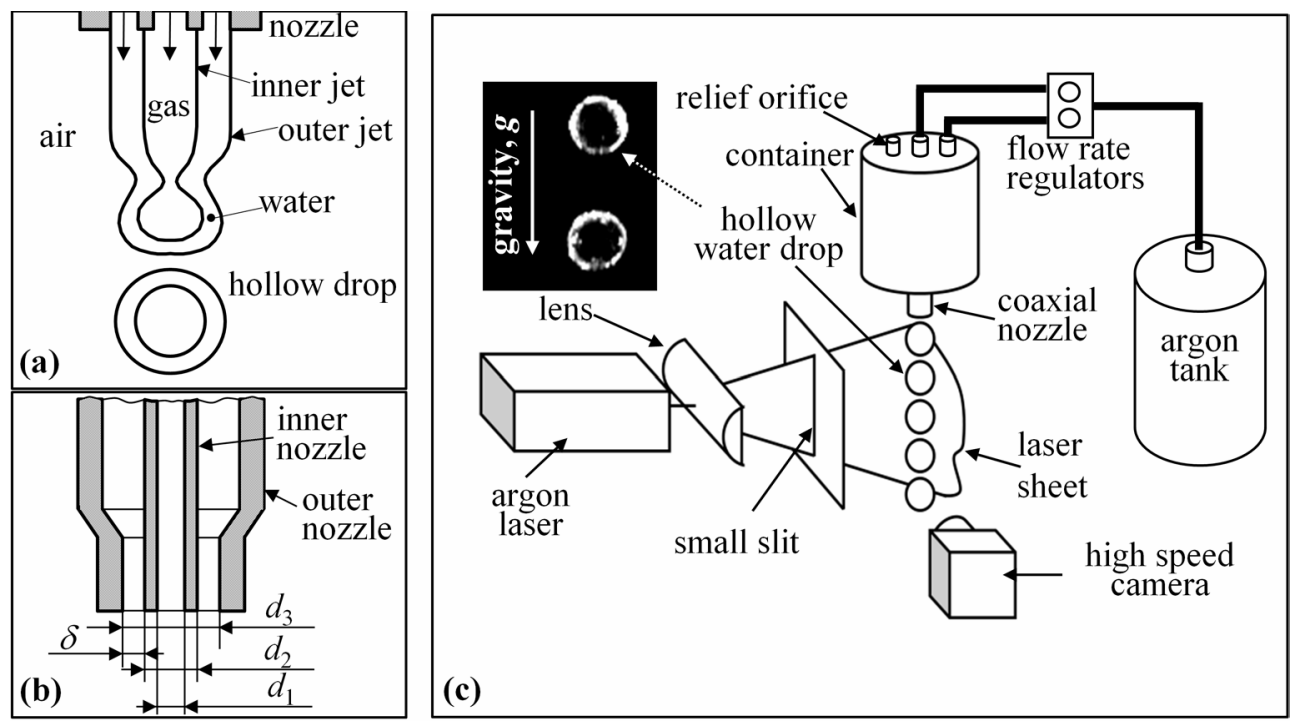

Fig. 1 Definition sketch: (a) a hollow jet, (b) cross-section view of a coaxial nozzle, and (c) schematic of apparatus for the formation of hollow water drops. 


$\begin{array}{llll}F r & : & \text { Froude number } \\ g & : & \text { gravity, } \mathrm{m} . \mathrm{s}^{-2} \\ L & : & \text { jet length, } \mathrm{m} \\ Q & : & \text { flow rate ratio } \\ R e & : & \text { Reynolds number } \\ t & : & \text { time, } \mathrm{s} \\ U & : & \text { velocity, } \mathrm{m}^{-1} \\ W e & : & \text { Weber number } \\ \rho & : & \text { density, kg.m }{ }^{-3} \\ \delta & : & \text { nozzle annular space, } \mathrm{m} \\ \mu & : & \text { dynamic viscosity, Pa.s } \\ \sigma & : & \text { surface tension, N.m }\end{array}$

\section{Subscript}

$$
\begin{array}{lll}
1 & : & \text { argon, internal wall of the inner nozzle } \\
2 & : & \text { water, external wall of the inner nozzle } \\
3 & : & \text { air, internal wall of the outer nozzle } \\
d & : & \text { drop detachment } \\
i & : & \text { in } \\
o & : & \text { out }
\end{array}
$$

\section{Experimentation}

\subsection{Experimental materials and nozzles}

We carry out experiments by employing water and argon as working materials, and two types of coaxial nozzles. The hollow jet with an inner jet of argon is formed in ambient air at rest and at room temperature. To clearly observe the formation as well as the hollowness of jets and drops, we seed water with hollow glass particles with a diameter on the order of $10 \mu \mathrm{m}$. The content of glass particles is about $1.5 \mathrm{~g}$ of glass/ $1 \mathrm{~kg}$ of water. When these particles are within a laser sheet they scatter laser light. Since glass particles are chemically neutral, surface tension and viscosity of water are essentially unchanged. Table 1 gives the properties of materials used in the present study. In order to investigate the effects of nozzle on the breakup patterns of hollow water jets, we use two types of coaxial nozzles. The cross-section of the coaxial nozzle is illustrated in Fig. 1 (b) in which $d_{1}, d_{2}$ and $d_{3}$ are the internal, external diameters of the inner nozzle and the internal diameter of the outer nozzle. The annular space of the coaxial nozzle is denoted as $\delta, \delta=\left(d_{3}-d_{2}\right) / 2$. The dimensions of the nozzles are given in Table 1.

\subsection{Apparatus description}

The apparatus for the experiments on the formation and breakup of a hollow water jet is depicted in Fig. 1 (c). It consists of a gas control system, a coaxial nozzle mounted to a

Table 1 Material properties and dimensions of coaxial nozzles

\begin{tabular}{cccc}
\hline Materials & Density, $\rho\left(\mathrm{kg} \cdot \mathrm{m}^{-3}\right)$ & Viscosity, $\mu(\mathrm{Pa} . \mathrm{s})$ & Surface tension, $\sigma\left(\mathrm{N} . \mathrm{m}^{-1}\right)$ \\
\hline Water & 1000 & 0.001 & 0.072 \\
Argon & 3.21 & 0.000023 & \\
Air & 1.29 & & $d_{3}(\mathrm{~m})$ \\
\hline Nozzle & $d_{1}(\mathrm{~m})$ & $d_{2}(\mathrm{~m})$ & 0.0015 \\
\hline Type I & 0.0008 & 0.001 & 0.0013 \\
Type II & 0.00055 & 0.0008 & \\
\hline
\end{tabular}


container, and an illumination system with a high speed camera (Photron FASTCAM 1024PCI) which is run at 6000 frames per second with a shutter speed of $1 / 6000 \mathrm{~s}$ for a resolution of $128 \times 1024$ pixels. Accordingly, the resolution of the digital images is in the range of $18-21$ pixels per one $\mathrm{mm}$. The typical outer size of hollow drops is about $3 \mathrm{~mm}$ and the outer jet length is greater than $6 \mathrm{~mm}$, as discussed below. Therefore, they can be measured with an error less than $5 \%$.

The most important part of the apparatus is the coaxial nozzle that consists of an inner nozzle inserted into the center of an outer nozzle, as shown in Fig. 1 (b). The gas control system is used to control the flow rate of argon gas directed to the inner nozzle of the coaxial nozzle. The water flow rate is controlled by pressurizing the container with argon gas from the gas system. The illumination system consists of an argon laser with a wavelength in the range of $333-529 \mathrm{~nm}$, which emits a laser beam with a diameter of $1-2$ $\mathrm{mm}$. A cylindrical lens expands the laser beam into a plane of about $1-2 \mathrm{~mm}$ in thickness that is narrowed by a slit of about $0.3 \mathrm{~mm}$ in width. The slit is placed close to the region of the drop formation (about $5 \mathrm{~cm}$ away from the center line of the drop train). The sheet of laser light cuts vertically hollow drops of water at their center. The camera views perpendicular to the laser sheet. The slit plays a very important role to give a clear observation of the hollowness of the jet as well as of hollow drops, as shown in Fig. 2. Without the slit almost all portions of the hollow jet and drops, which are illuminated, fall in the laser plane. Therefore the hollowness could not be identified, as shown in Fig. 2 (a). However, we can observe the hollowness by using the slit to narrow the laser plane to a sheet of less than $0.5 \mathrm{~mm}$ in thickness, as shown in Fig. 2 (b).

\subsection{Experimental parameters}

The formation and breakup of a hollow jet is affected by a large number of parameters such as the properties of hollow jet fluids, flow rates, and the nozzle size. Accordingly, a large number of dimensionless parameters ${ }^{(13)}$ characterize the fluid dynamics of the hollow jet. Here, we consider a few parameters as follows

$\begin{array}{ll}\text { Weber numbers } & W e=\rho_{2} U_{2}^{2} \delta / \sigma \\ \text { flow rate ratio } & Q=\frac{d_{1}^{2} U_{1}}{\left(d_{3}^{2}-d_{2}^{2}\right) U_{2}} \\ \text { Reynolds number } & \operatorname{Re}=\rho_{2} U_{2} \delta / \mu_{2} \\ \text { Froude number } & F r=U_{2}^{2} /(g \delta) \\ \text { density ratios } & \rho_{1} / \rho_{2} ; \rho_{3} / \rho_{2} \\ \text { viscosity ratio } & \mu_{1} / \mu_{2}\end{array}$

$\mu_{1} / \mu_{2}$

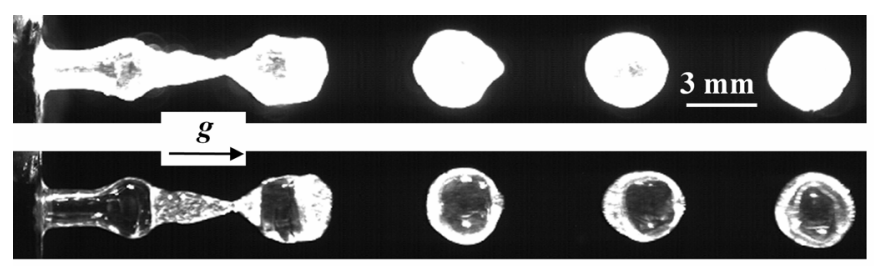

(a)

without the slit

Fig. 2 Formation of hollow jets and drops: (a) without the presence of the slit and (b) with the presence of the slit. $W e=6.74, Q=1.1, \operatorname{Re}=348, F r=792, D=1.5$. Scale bar applies to (a) and (b). 
diameter ratio

$$
D=d_{3} / d_{2}
$$

Here, the subscripts 1,2 and 3 of density $\rho$, viscosity $\mu$ and velocity $U$ indicate argon, water and air, respectively. $U_{1}$ and $U_{2}$ are the average argon gas and water velocities at the nozzle exit, respectively. $g$ is the gravity, $g=9.8 \mathrm{~m} . \mathrm{s}^{-2}$.

The choice of the nondimensional parameters follows Kendall ${ }^{(19)}$ and Chen and Lin ${ }^{(13)}$. Since we use water and argon for all experimental runs, the density and viscosity ratios are unchanged, i.e. $\rho_{1} / \rho_{2}=0.00321, \rho_{3} / \rho_{2}=0.00129, \mu_{1} / \mu_{2}=0.023$. Other parameters are given in Table 2. The ranges of We, $Q$, Re and Fr correspond to the bubble formation regime ${ }^{(20)}$. Because of our purpose of identifying different final breakup patterns of the hollow water jet and of the great importance of capillary effects on the process, the two most important parameters are $W e$ and $Q$. However, $R e$ and $F r$ are also addressed to justify our observations.

\section{Results and discussion}

In the present study, we investigate the formation and final breakup of a hollow water jet by videographic and photographic techniques. We focus on the regime of bubble formation in which various final breakup patterns of the hollow water jets are identified.

Table 2 Ranges of the investigated parameters in the present study

\begin{tabular}{llllllll}
\hline Nozzle & $U_{1}\left(\mathrm{~m}_{\mathrm{s}}^{-1}\right)$ & $U_{2}\left(\mathrm{~m} \cdot \mathrm{s}^{-1}\right)$ & $W e$ & $Q$ & $R e$ & $F r$ & $D$ \\
\hline Type I & $0.33-18.25$ & $1.31-2.06$ & $5.94-14.67$ & $0.08-5.19$ & $327-514$ & $698-1724$ & 1.5 \\
Type II & $0.85-42.46$ & $1.56-2.14$ & $8.42-15.95$ & $0.09-5.84$ & $389-536$ & $989-1875$ & 1.625 \\
\hline
\end{tabular}

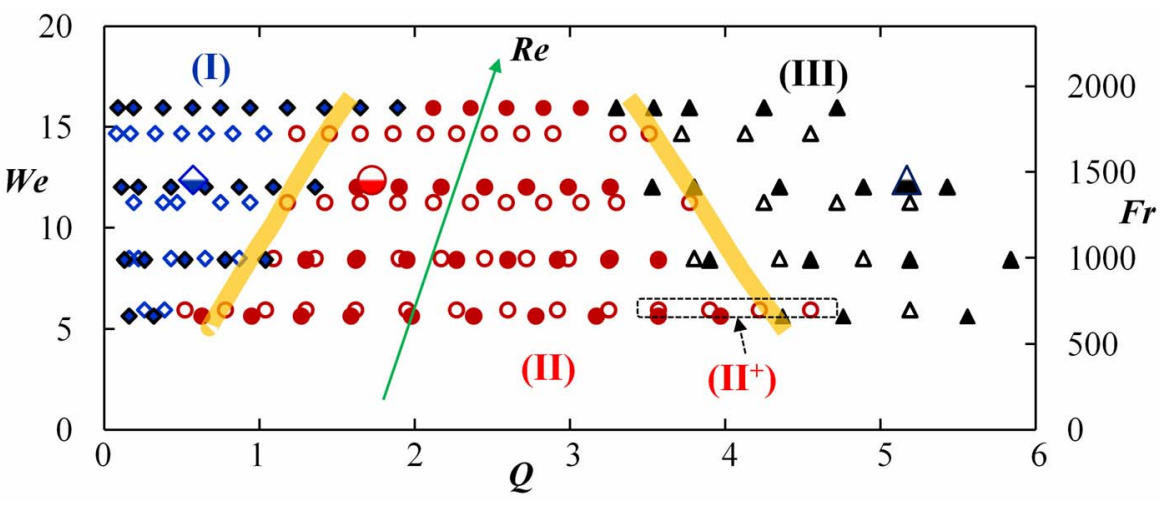

Fig. 3 Map of breakup patterns of hollow water jets. Diamonds, circles and triangles represent pattern (I) - mixed hollow and simple drop formation, pattern (II) - single-core hollow drop formation and pattern (III) - multi-core hollow drop formation, respectively. Open and solid symbols represent the nozzle type I $(D=1.5)$ and the nozzle type II $(D=1.625)$, respectively. Pattern $\left(\mathrm{II}^{+}\right)$also belongs to pattern (II). Three large half-filled symbols represent $W e$ and $Q$ for these three patterns observed in Kendall's work ${ }^{(19)}$ (Re in Ref. (19) is higher than the current data). Orange lines roughly separate different patterns.

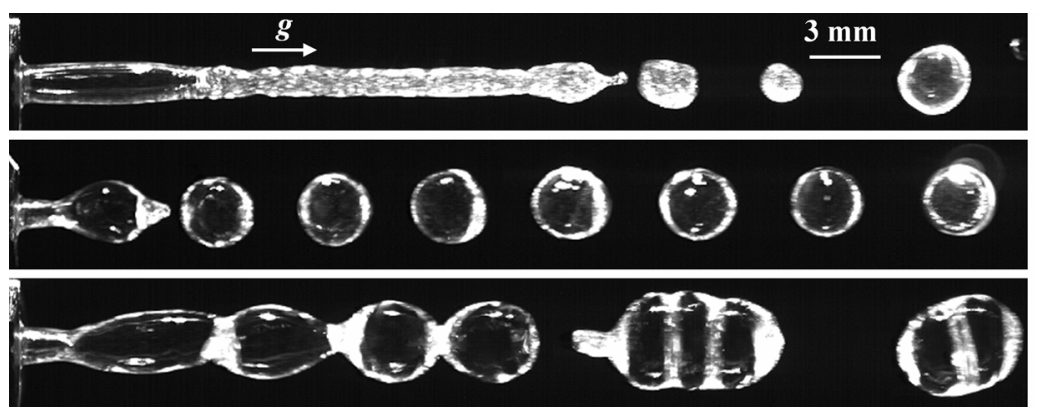

(a) $\mathrm{We}=8.5$

$Q=0.22, R e=391$ $F r=997, D=1.5$

(b) $W e=5.63$ $Q=3.17, \operatorname{Re}=318$ $F r=662, D=1.625$

(c) $W e=11.26$ $Q=5.19, R e=450$ $F r=1323, D=1.5$

Fig. 4 Breakup patterns: (a) pattern (I), (b) pattern (II), and (c) pattern (III). Scale bar applies to (a) - (c). 


\subsection{Breakup patterns of a hollow jet}

We employ two types of coaxial nozzles with diameter ratios $D=1.5$ and 1.625 (Tables 1 and 2). For each nozzle type, we observe the formation and final breakup of the hollow jet at four Weber numbers, We, and various gas-to-water flow rate ratios, $Q$. Following the observations, we group the final breakup of the hollow water jets into three patterns in a We - $Q$ parameter space: (I) mixed hollow and simple drop formation, (II) single-core hollow drop formation, and (III) multi-core hollow drop formation, as shown in Fig. 3. Figure 4 shows typical examples illustrating three patterns of the final breakup.

Although Kendall ${ }^{(19)}$ did not classify his hollow jets into different patterns, we can identify three patterns described herein in Fig. 7 in Ref. (19). Based on the physical parameters for his cases in Fig. 7, the important dimensionless parameters of those cases for comparison with our work are $W e=12.38$ and $Q=0.57$ (left photograph, Fig. 7, Ref. (19)), $Q=1.72$ (middle photograph, Fig. 7, Ref. (19)), $Q=5.17$ (right photograph, Fig. 7, Ref. (19)). We map these points in our $W e-Q$ parameter space, as shown in Fig. 3. Figure 3 shows that our work agrees well with Kendall's work ${ }^{(19)}$.

\subsection{Mixed hollow and simple drop formation - pattern (I)}

According to Fig. 3, pattern (I) occurs at all Weber numbers and low flow rate ratios. Under these conditions, the inner jet of gas breaks up closer to the nozzle than the water outer jet does, but at relatively long temporal intervals. Thus inner gas bubbles detaching from the gas jet travel downstream in the jet of water. At a relatively low flow rate ratio, simple water drops are formed in a fashion similar to a water drop detaching from a single water jet, as shown in Fig. 4 (a). When the flow rate ratio increases, a ligament of water is formed between two main hollow drops, as shown in Fig. 5 (a). The ligament breaks first at its downstream end that connects the leading hollow drop, and then at its upper end that connects the trailing drop. This ligament generates simple water drops during retraction process made by surface tension ${ }^{(21)}$. Here hollow satellite drops are also observed (Fig. 5 (b)).

Figure 6 shows the variation of the dimensionless length of the outer jet, $L_{\mathrm{o}} / d_{3}$ (see Fig. 5 (b) for the definition of $L_{o}$ ), with time measured forward from the moment when a hollow drop just detaches $t-t_{d}$. As seen in that figure, the general pattern is a rise to a certain

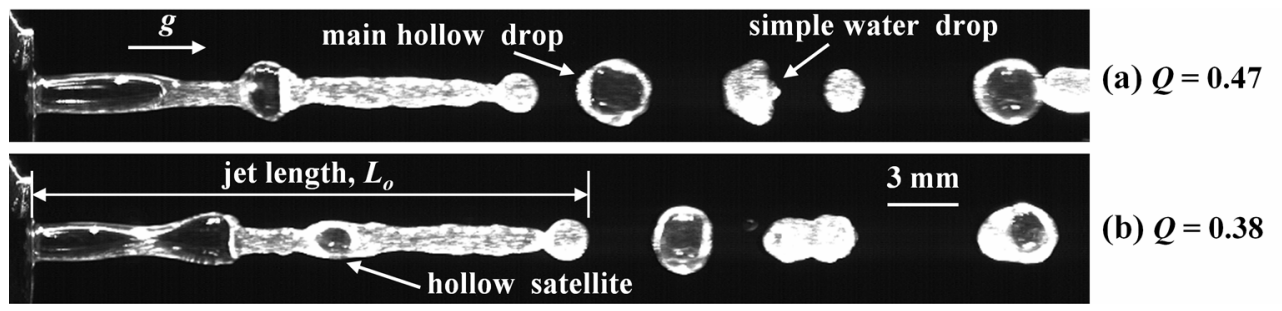

Fig. 5 Mixed hollow and simple drop formation (pattern (I)): (a) simple water drops formed from the ligament between two main hollow drops, and (b) hollow satellite drop formation. Other parameters: $W e=$ 11.26, $\operatorname{Re}=450, F r=1323, D=1.5$. Scale bar applies to (a) and (b).

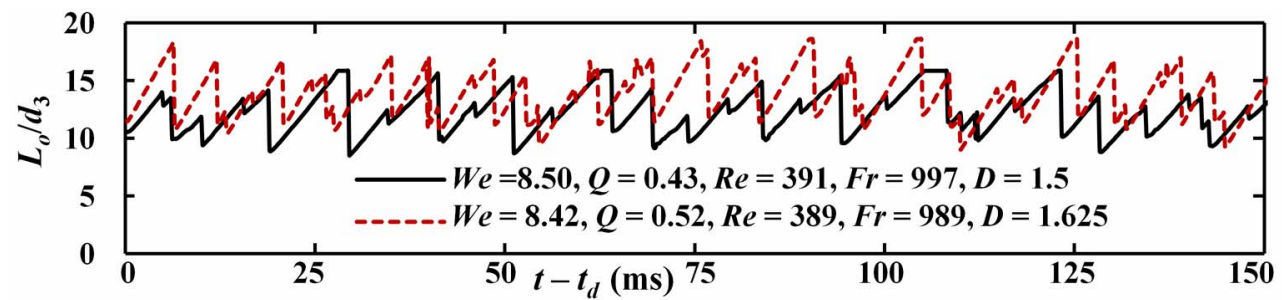

Fig. 6 Time dependence of the dimensionless length of the outer jet, $L_{o} / d_{3}$, in pattern (I). In Figs. 6 , 8 and $10, t_{d}$ is the time at the instant of drop detachment. 

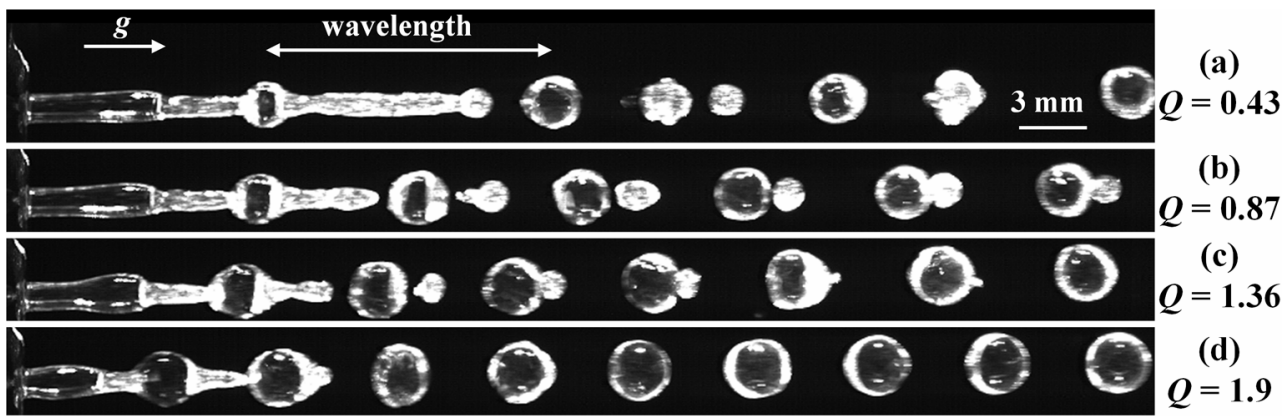

Fig. 7 Simple drop suppression by increasing the flow rate ratio. $W e=12.02, \operatorname{Re}=465, F r=1413, D=$ 1.625. (a) - (c) pattern (I), and (d) pattern (II). Scale bar applies to (a) - (d).

length, detachment of a drop, with a build up in length to form the next drop. This results in a sawtooth pattern ${ }^{(22)}$. However, as shown in Fig. 6 , the jet length is not periodic, but rather chaotic due to the formation of simple water drops. Figure 6 also makes plain that the simple drop formation results in non-uniformity in the size of hollow drops. Therefore, for encapsulation applications ${ }^{(1,2)}$ we must avoid producing hollow drops in pattern (I). Figure 3 makes plain that we can suppress pattern (I) by increasing the gas-to-water flow rate ratio. It is more evident by considering Fig. 7. At a low flow rate ratio (Fig. 7 (a)), the wavelength is rather long, and a long ligament yields large simple water drops. Increasing the flow rate ratio results in decreasing the wavelength ${ }^{(20)}$, and in shortening the ligament. Thus the size of simple drop formed from the ligament decreases. The simple drop merges backward with the adjacent main hollow drop in about four wavelengths, as shown in Fig. 7 (b). When we continuously increase $Q$, it merges backward in about two wavelengths (Fig. 7 (c)). As a result, at a higher $Q$, the hollow jet breaks up in pattern (II) in which there are not simple water drops (Fig. 7 (d)). Thus it is evident that the formation of pattern (I) is basically due to the insufficient flow rate of the inner gas jet. The simple water drops in Figs.7 (b) and (c) are considered as simple satellite drops. However if the flow rate ratio is too high, the breakup of the hollow jet will be in pattern (III). Figure 3 further shows that pattern (I) widens as $W e$ increases because increasing $W e$ promotes the jetting modes ${ }^{(23)}$ in which irregularity in the drop formation is dominant ${ }^{(17)}$.

\subsection{Multi-core hollow drop formation - pattern (III)}

Pattern (III), multi-core hollow drop formation, occurs at high flow rate ratios, as shown in Fig. 3. Two or more gas cores accumulate in a large water shell, as shown in Fig. 4 (c). The inner gas jet produces gas bubbles at sufficiently short temporal intervals ${ }^{(19)}$. Therefore, they remain interconnected until the outer jet breaks up into a large drop which encapsulates a few gas cores because of surface tension. The multi-core hollow drop can contain two or three gas cores (Fig. 4 (c)). Even a drop enclosing up to six gas cores can be formed, as shown in Fig. 8 (a). However, it is difficult to produce a large number of gas cores in a large water shell since the annular space $\delta$ is thin $(D=1.5$ and 1.625). In contrast, a compound drop that contain many inner drops can easily be generated by forming a compound jet of very viscous liquids ${ }^{(17)}$ since it is easier to encapsulate and control the breakup of a jet of more viscous fluids ${ }^{(13)}$. Similarly to pattern (I), the formation of multi-core hollow drops is very irregular. Figure 8 (b) shows chaos in the time dependence of the outer jet length in pattern (III). Figure 8 (b) also makes plain that the hollow drops formed in pattern (III) are definitely non-uniform, as seen from in Figs. 4 (c) and 8 (a). In addition to non-uniformity in size, we observe that some multi-core hollow drops burst because in comparison with single-core hollow drops, they contain a larger volume of gas, and thus experience a larger aerodynamic drag force as they fall in air whereas their wall is relatively thin. Furthermore, pattern (III) is close to the regime of annular jet formation ${ }^{(20)}$ 
in which the wavy jet surface and segmented thin liquid columns are dominant. It is observed that the gas cores can merge to form a large single-core hollow drop further downstream. However, this merging can sometimes destroy the multi-core hollow drop. For encapsulation applications in which uniform-sized hollow drops are desired, pattern (III) must be avoided.

\subsection{Single-core hollow drop formation - pattern (II)}

Since, as mentioned previously, we have interest in encapsulation applications ${ }^{(1,2)}$, we will focus much more on pattern (II). As shown in Fig. 3, the formation of single-core hollow drops can be found at $Q$ in the range of $0.39-4.55$. When $W e$ increases, pattern (II) narrows. In this pattern, we further investigate how the flow rate ratio $Q$ affects the size and wall thickness of hollow water drops, and the formation frequency. However, the effects of the flow rate ratio on the breakup lengths of both inner and outer jets extend to patterns (I) and (III).

Utada et al. ${ }^{(17)}$ reported that double emulsions (compound drops) enclosing one inner drop can be generated when both inner and outer jets are simultaneously dripping or simultaneously jetting. Dripping is characterized by drops detaching close to the nozzle exit, within one or two nozzle diameters $d_{3}$. In contrast, jetting produces drops far from the nozzle exit with a breakup length of more than three times the nozzle diameter $d_{3}$. In the present study, we find that single-core hollow drops can be produced when the inner jet of gas is dripping and the outer jet of water is either dripping (Fig. 4 (b)) or jetting (Fig. 9). Figure 9 shows the breakup process in one cycle. We can see here that the formation of the gas core is similar to that found in Kendall's work ${ }^{(19)}$ (see Fig. 3 in Ref. (19)), in which he described in detail how the gas core was produced. The velocity ratio of gas to water for the

(a)
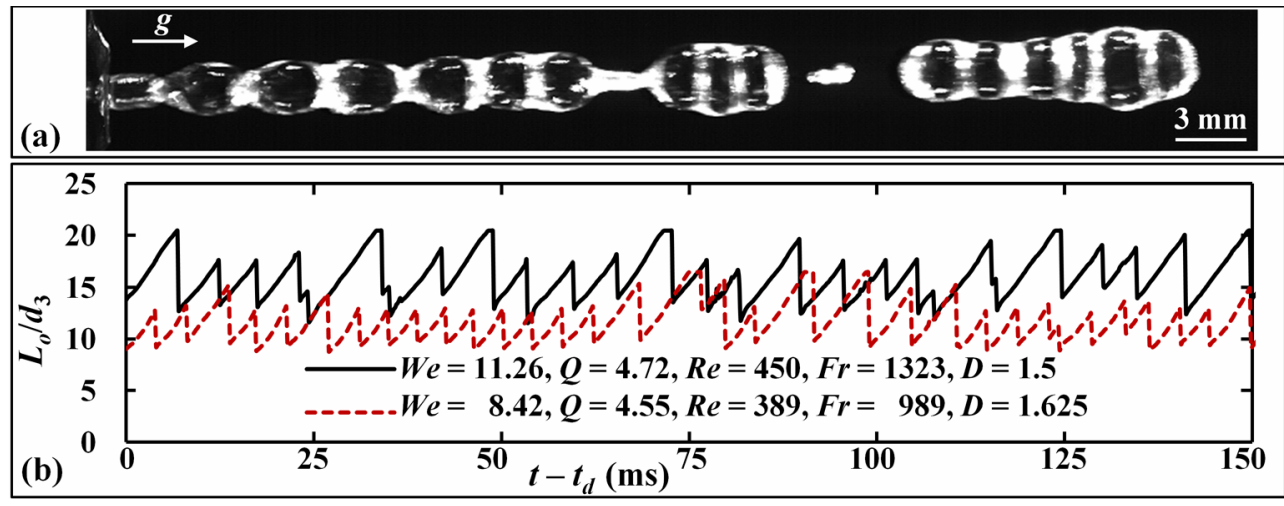

Fig. 8 Multi-core hollow drop formation (pattern (III)): (a) formation of a six-core hollow drop, We = 15.95, $Q=4.72, \operatorname{Re}=536, F r=1875, D=1.625$ and (b) time dependence of the dimensionless length of the outer jet, $L_{o} / d_{3}$.

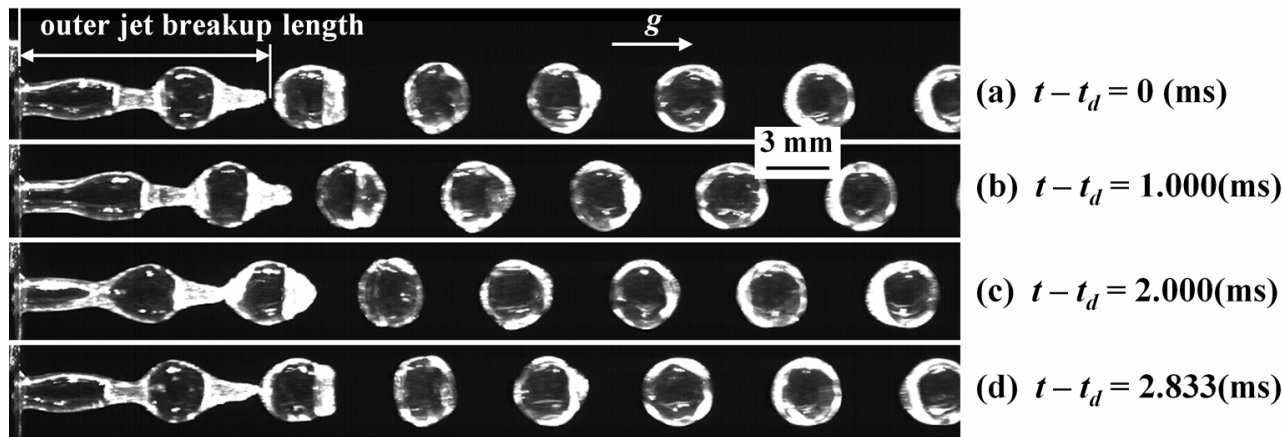

Fig. 9 A cycle of hollow drop formation in pattern (II). $W e=14.67, Q=2.07, \operatorname{Re}=514, F r=1724, D=$ 1.5. Scale bar applies to (a) $-(d)$. 


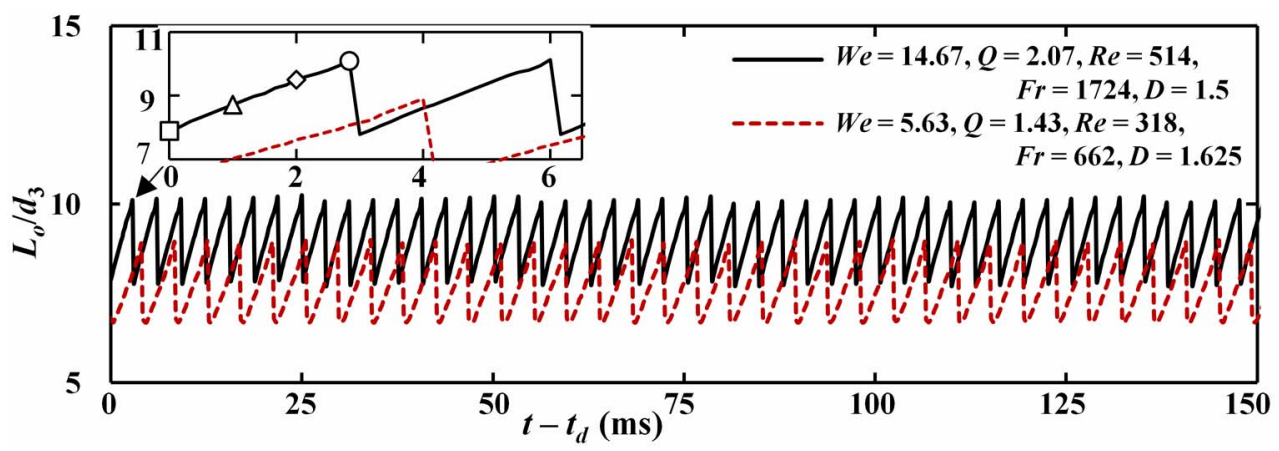

Fig. 10 Time dependence of the dimensionless length of the outer jet, $L_{o} / d_{3}$, in the pattern (II). Open rectangle, triangle, diamond and circle correspond to Figs. 9 (a), (b), (c) and (d), respectively.

case in Fig. 9 is 4.03 whereas that in Kendall's case is three. Similarly to the results found in Ref. (19), Fig. 9 further shows that the formation of hollow drops in pattern (II) is highly periodic. The periodicity is shown more evidently in Fig. 10 which shows the sawtooth patterns of the outer jet length varying with time. This formation (pattern (II)) is totally different from those seen in Figs. 6 (pattern (I)) and 8 (b) (pattern (III)) where chaos is dominant. Figure 10 also makes plain that uniform-sized hollow drops can be generated in pattern (II) (Fig. 9) without introduction of external periodic excitation. This pattern makes the hollow jet profoundly different from a single jet with regard to the uniformity in size of drops ${ }^{(19)}$ since it is difficult to produce uniform-sized drops from a single jet in a jetting mode without external excitation. For compound jets under the external periodic perturbations, Chiu and Lin ${ }^{(18)}$ found a range of uniform-sized compound drops for each core-to-shell mass ratio in terms of a ratio of disturbance wave length to undisturbed diameter of the compound jet. In stead, we find a spectrum of uniform-sized hollow drops in the $W e-Q$ space using two types of nozzle: $D=1.5$ and $D=1.625$, as shown in Fig. 3 .

However, a profound difference from those found in previous studies is that single-core hollow drops can be formed by either merging of two gas cores (bubble) or reunion of the gas jet with a just formed gas bubble before the breakup of the outer jet, as shown in Fig. 11. At the beginning of the sequence, as seen from Fig. 11 (a), a hollow drop with one gas core has just detached from the jet in which the first gas bubble has been formed. A second gas bubble is growing from the gas jet because of continuous feeding of gas at the inner nozzle exit. As time advances, the gas jet elongates, and tends to merge with the first gas bubble (Fig. 11. (b)). Merging and breakup then occur to form an elongated bubble (Fig. 11 (c)). The unbalanced force of surface tension makes this elongated bubble spherical (Fig. 11 (d)). As a result, a newly single-core hollow drop is about to be formed (Fig. 11 (e)). A notable feature of this process is the formation of a vertical water jet inside the gas bubble after merging, as shown in Fig. 11 (d). This phenomenon is similar to the formation of a water jet in busting of gas bubbles at a free water surface ${ }^{(24)}$. It appears that the merging of gas bubbles and the formation of the water jet in the newly formed gas bubble has not been reported in the literature.

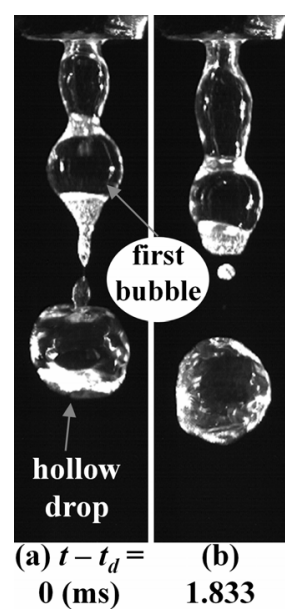

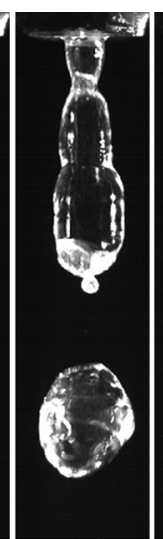

(c)
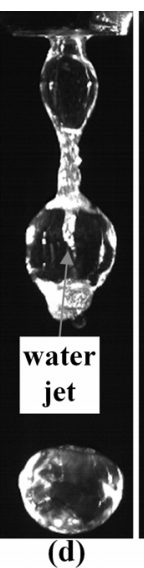

5.666

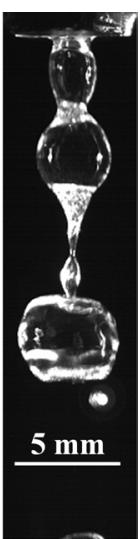

(e)

9.666
Fig. 11 Formation of a single-core hollow drop by merging of gas bubbles before detachment of a water shell in pattern $\left(\mathrm{II}^{+}\right)$. We $=5.94, Q=3.57, \mathrm{Re}=327, \mathrm{Fr}=$ $698, D=1.5$. Scale bar applies to (a) - (e). 
Because of merging, the single-core hollow drops formed in this way are larger than those discussed previously, and experience a large deformation after breakup (Fig. 11). However, they reach a spherical shape downstream due to surface tension. As shown in Fig. 11, a tinny water satellite drop is formed between the hollow drop and the hollow jet. Because of this irregular formation, we classify it into pattern $\left(\mathrm{II}^{+}\right)$. Pattern $\left(\mathrm{II}^{+}\right)$occurs at $W e=5.94, Q$ $=3.57-4.55, D=1.5$, as shown in Fig. 3. However, this pattern does not occur in the range of We investigated for the nozzle type II $(D=1.625)$. It would occur at a lower We. However, we have not investigated at such a $W e$ because the formation of pattern $\left(\mathrm{II}^{+}\right)$is not the main purpose of our present study. Furthermore, it is observed that pattern $\left(\mathrm{II}^{+}\right)$is sensitive to the coaxiality of the coaxial nozzle. Here we just mention it as a newly observed feature in the fluid dynamics of a hollow jet. Therefore, we group it into a general pattern, pattern (II), since the final breakup of the hollow jet in pattern $\left(\mathrm{II}^{+}\right)$is also the formation of a single-core hollow drop, as shown in Fig. 11. However, in order to distinguish pattern $\left(\mathrm{II}^{+}\right)$ among others, we mark it with $\left(\mathrm{II}^{+}\right)$in some figures. The formation of pattern $\left(\mathrm{II}^{+}\right)$affects considerably the formation frequency and size of hollow drops, as discussed below.

Next, we consider the effects of the flow rate ratio $Q$ on the size and shell thickness of hollow drops, and the formation frequency $f$ in the single-core hollow drop formation, pattern (II). It is reported that the gravity is unimportant to the hollow drop formation ${ }^{(19)}$. Therefore, in the following, we do not address the Froude number.

Figure 12 shows the $Q$ dependence of the formation frequency $f$ in pattern (II). According to the figure, as $Q$ increases, the frequency increases. This trend agrees with that found by Kendall ${ }^{(19)}$. Moreover, Kendall ${ }^{(19)}$ reported that the formation frequency varies with a -1.5 power of the nozzle size. Accordingly, under best operation with water, the frequencies for the nozzle types I $\left(D=1.5, d_{3}=1.5 \mathrm{~mm}\right)$ and II $\left(D=1.625, d_{3}=1.3 \mathrm{~mm}\right)$ would be about 380 and $490 \mathrm{~Hz}$, respectively. Figure 12 confirms the law. Additionally, as seen from Fig. 12, at $W e=8.5, R e=391, D=1.5$ (type I), the frequency ranges from 183 to $258 \mathrm{~Hz}$. According to the law ${ }^{(19)}$, the frequency for the nozzle type II at nearly equal Reynolds and Weber numbers $(W e=8.42, R e=389, D=1.625)$ would be $230-320 \mathrm{~Hz}$ while it is $280-430 \mathrm{~Hz}$ (Fig. 12). Evidently, we find good agreement with the law. Figure 12 also makes plain that increasing $W e$ and $R e$ results in increasing the frequency. As mentioned previously, the formation frequency of hollow drops in pattern $\left(\mathrm{II}^{+}\right)$appeared at $W e=8.5, R e=327$ and $D=1.5$ is affected by merging of gas bubbles (Fig. 11), and thus lower than that in pattern (II), as seen in Fig. 12.

Next, concerning the outer diameter of the hollow drop, it is reported to be approximately twice the internal diameter $d_{3}$ of the outer nozzle ${ }^{(1,19)}$. In our experiments, the average outer diameter $d_{o}$ is from $1.9-2.5$ times $d_{3}$, as shown in Fig. 13 (a). The measured diameters in Fig. 13 (a) are average values. Typically twenty to thirty hollow drops are taken for measurements. For each drop, we first track the outer interface of the drop, and then calculate an equivalent outer diameter. As mentioned previously, the hollow

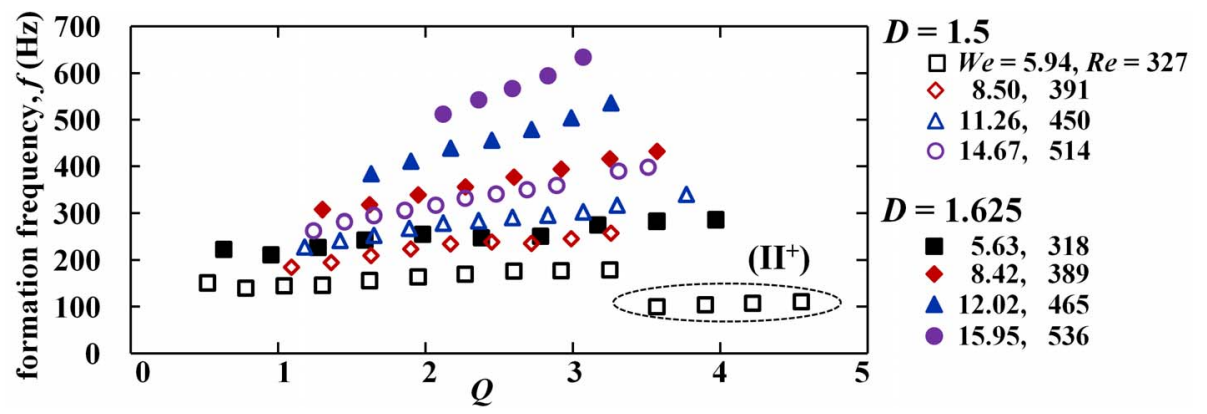

Fig. 12 Variation of formation frequency of hollow drops with the flow rate ratio, $Q$, in pattern (II). Open and solid symbols represent $D=1.5$ and $D=1.625$, respectively. Symbol definitions in Fig. 12 are also used for Figs. 13 and 14. 
drops in pattern $\left(\mathrm{II}^{+}\right)$are larger due to the merging of gas bubbles before pinch-off of the outer (water) jet (Fig. 11). Accordingly, the outer diameter of these drops is about $3.2 d_{3}$ (Fig. 13 (a)). Figure 13 (a) further indicates that an increase in $Q$ leads to a slight increase in the outer diameter of the hollow drop.

Although the use of the laser system provides clear observations of the formation and breakup of water jets as well as their hollowness, the photography fails to bring out details of the shell wall thickness. Alternatively, using the average outer diameters $d_{o}$ of the drops, the formation frequency $f$, the size of the coaxial nozzle $d_{2}$ and $d_{3}$, the gas and water flow rates, and the mass conservation, the inner diameter $d_{i}$ of the hollow drop can be calculated:

$$
d_{i}=\sqrt[3]{d_{o}^{3}-3\left(d_{3}^{2}-d_{2}^{2}\right) U_{2} /(2 f)}
$$

Figure 13 (b) shows the variation with $Q$ of the wall thickness of the hollow drop. The figure confirms that an increase in $Q$ results in a decrease in the wall thickness ${ }^{(19)}$. Combining data in Figs. 4 and 6 in Ref. (19), we find that when the flow rate ratio increases from 1.08 to 2.82 , the shell wall thickness nondimensionalized by the annular space $\delta$ of the coaxial nozzle decreases from 0.9 to 0.5 in Kendall's work ${ }^{(19)}$. In our investigation, the wall thickness varies from 0.5 to 1.5 times $\delta$ as $Q$ varies in the range of $0.52-4.55$, as shown in Fig. 13 (b).

In the following, we investigate the effect of $Q$ on the hollow jet breakup lengths. As mentioned previously, the formation frequency, the diameter and the shell wall thickness are measured and calculated in pattern (II). However, the breakup lengths are measured for all patterns in order to give the detailed effects of the flow rate ratio in the bubble formation
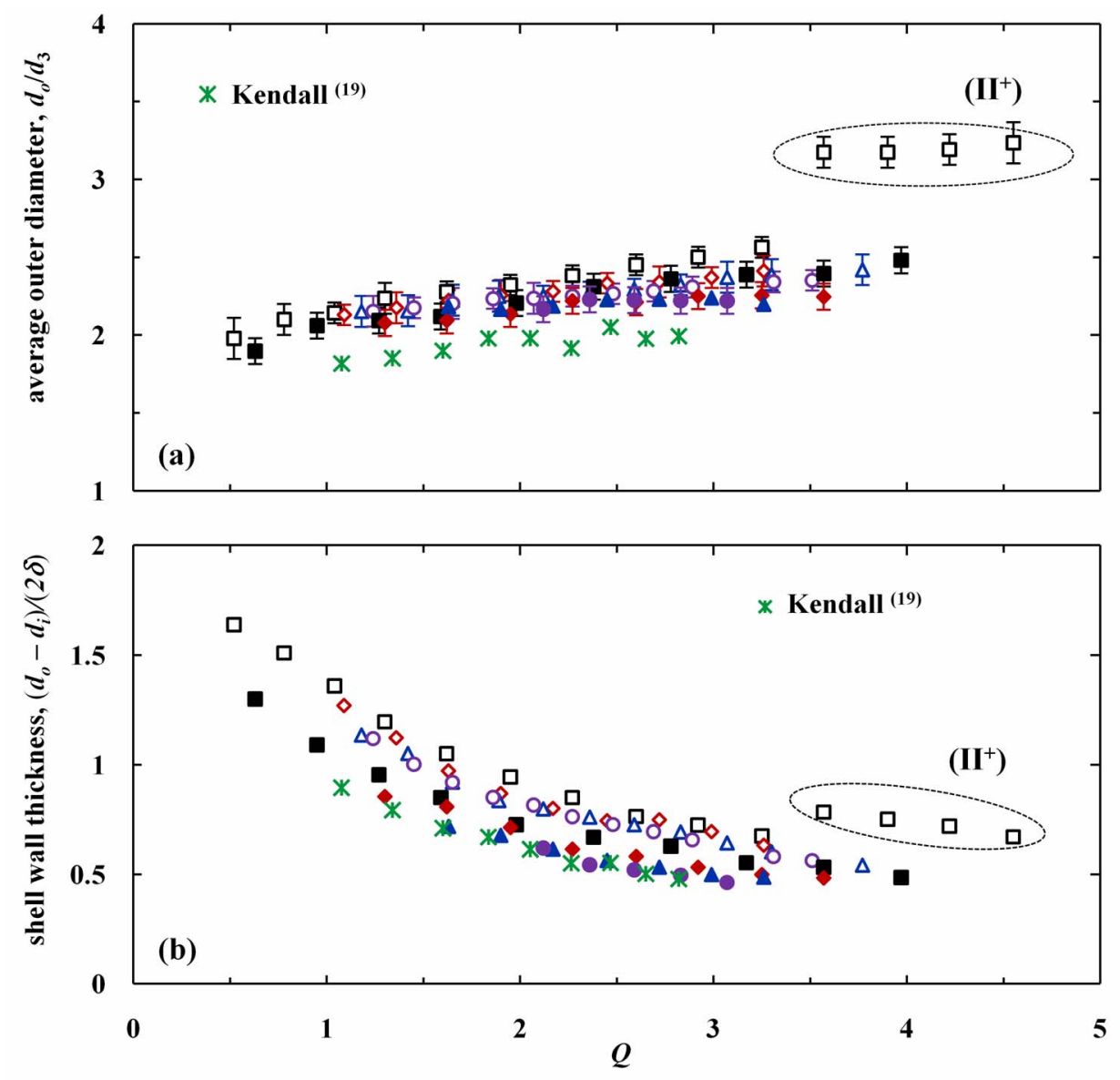

Fig. 13 Variation with the flow rate ratio, $Q$, of (a) the dimensionless average outer diameter of hollow drops and (b) the shell wall thickness in pattern (II). Open and solid symbols represent $D=1.5$ and $D=$ 1.625, respectively. Other symbol definitions as in Fig. 12. 
regime. Using the present technique, as described in the previous section, we can easily measure the breakup lengths of both inner (gas) and outer (water) jets. The breakup length of the outer jet (Fig. 9 (a)) used in the following is defined as the outer jet length (Fig. 5 (b)) at the instant just after hollow drop detachment. Similarly, the breakup length of the inner jet is the length of the inner jet (measured from the nozzle exit) at the instant just after gas core pinch-off. In addition, the breakup lengths of the inner and outer jets are average values by using typically from five to ten photographs for the measurements since the evolution in time of the jet in patterns (I) and (III) is chaotic, as shown in Figs. 6 and 8 (b).

Figure 14 shows the effect of $Q$ on the breakup lengths of the hollow jet for two nozzle types. As shown in Fig. 14 (a), the breakup length of the inner jet decreases as $Q$ increases. In contrast, the breakup length of the outer jet behaves complexly towards an increase in $Q$ (Fig. 14 (b)). Generally, it first decreases as $Q$ increases from pattern (I) to pattern (II), and reaches a minimal in pattern (II). When $Q$ continuously increases, it then increases with $Q$ from pattern (II) to (III). Although Kendall ${ }^{(19)}$ did not investigate in detail the effect of $Q$ on the breakup lengths, we can see a similar trend by considering his photographs (see Fig. 7 in Ref. (19)). This variation can be seen more obviously in Fig. 15. However, the variation of the outer jet breakup length with $Q$ in the present study and that seen from Kendall's photographs are different from that found by $\mathrm{Li}$ and Shen ${ }^{(20)} \mathrm{Li}$ and Shen ${ }^{(20)}$ stated that in the bubble formation regime, the jet breakup length decreases as the air-to-water velocity ratio increases. The difference between our results and those found by Li and Shen ${ }^{(20)}$ may be caused by the fact that their results were obtained from conditions at which the inner and outer jets broke up almost simultaneously (see Fig. 3 in Ref. (20)). In addition, they conducted experiments for four water velocities at each of which two to four gas velocities were carried out. Therefore, their results may not cover pattern (I) and (III). Moreover, because of the simultaneous breakup, the variation of the outer jet breakup length with respect to the velocity ratio followed that of the gas jet. It explains why their results agreed fairly with linear analysis ${ }^{(20)}$. In the present study, we find a similar trend for the inner gas jet (Fig. 14 (a)). However, our results that cover three patterns go far beyond the linear theory ${ }^{(12,13)}$. Figure 14 also makes plain that the breakup lengths increase as $W e$ and $R e$ increase. Increase in the breakup length due to an increase in We agrees with the prediction of linear theory ${ }^{(13)}$. The increase of the outer jet breakup length with $R e$ is understandable because increasing $R e$ corresponds to increasing the inertia that carries the outer jet downstream. Because we increase $R e$ by increasing the water velocity, the water viscous
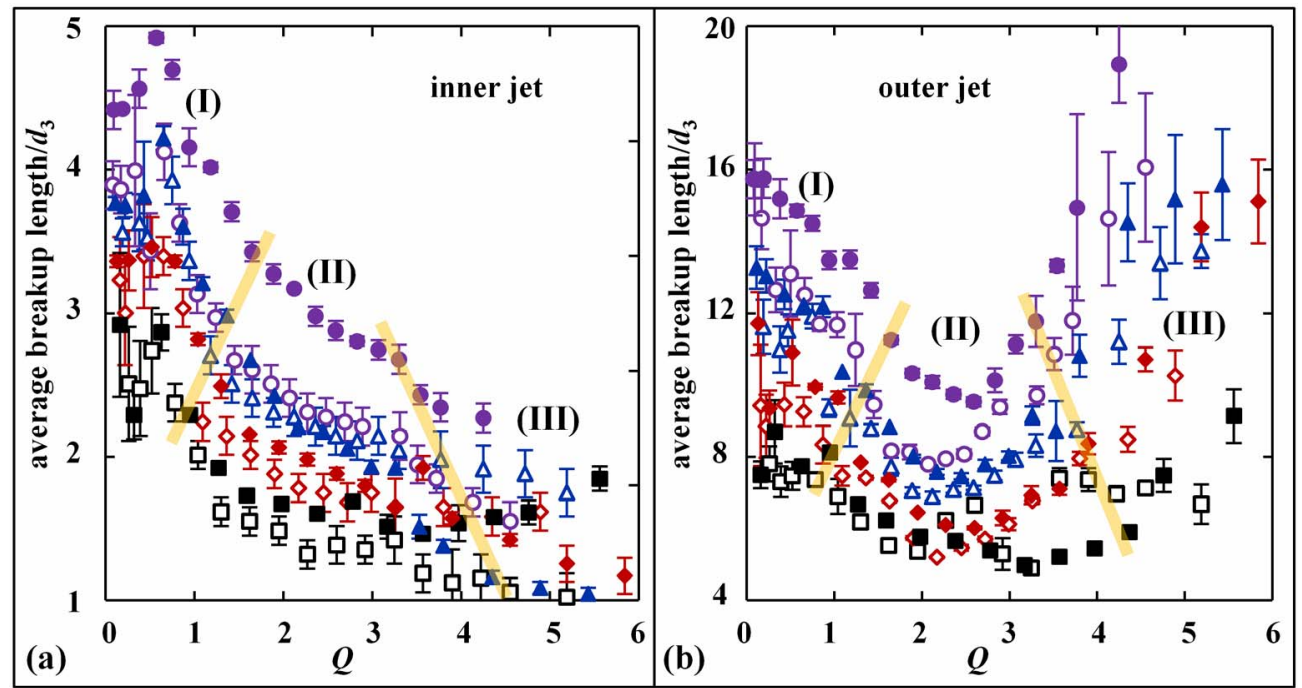

Fig. 14 Variation with the flow rate ratio of the sample averge breakup lengths of (a) the inner jet of gas and (b) the outer jet of water. Open and solid symbols represent $D=1.5$ and $D=1.625$, respectively. Other symbol definitions as in Fig. 12. Orange lines roughly separate different patterns. 


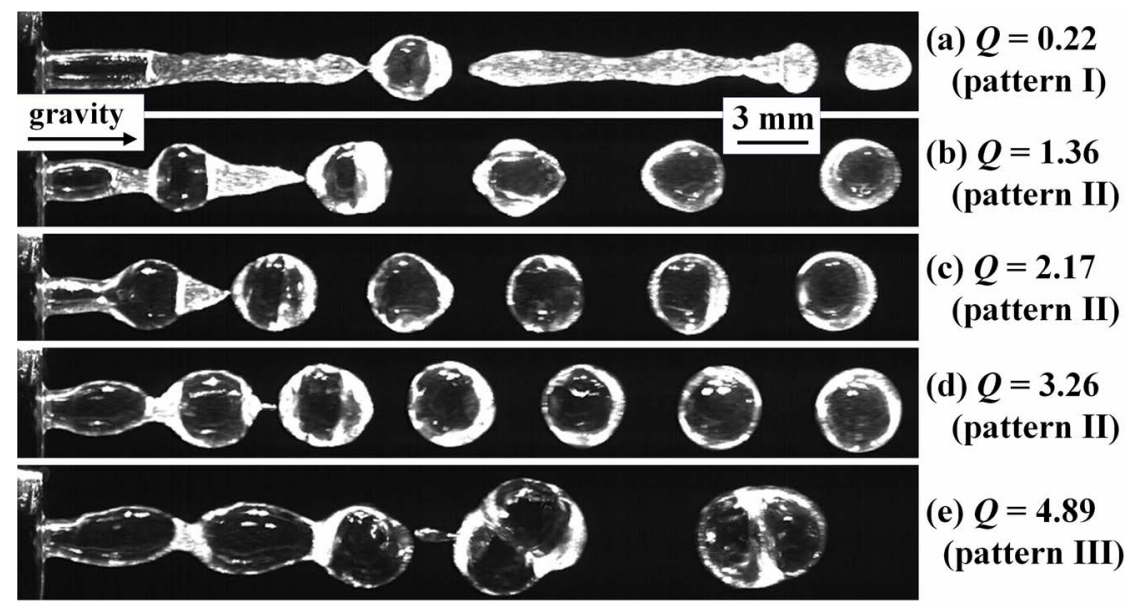

Fig. 15 Effects of the flow rate ratio, $Q$, on the outer jet breakup length. $W e=8.5, R e=391, F r=997, D$ $=1.5$. Scale bar applies to (a) $-(\mathrm{e})$.

drag that pulls the growing bubble of the inner gas jet is also increased. Therefore, the breakup length of the inner jet increases with $R e$. However, this effect is not evident since water viscosity is low. In addition to showing the variation of the outer jet breakup length with $Q$, Fig. 15 also confirms that increasing $Q$ results in slightly increasing the outer diameter of the hollow drop in pattern (II), as shown in Figs. 15 (b)-(d).

\section{Conclusions}

We have experimentally studied the formation and breakup of a hollow water jet using two nozzle types with diameter ratios $D=1.5$ and $D=1.625$. Following our observations, three final breakup patterns of hollow jets are identified in the Weber number versus flow rate ratio parameter space, $W e-Q$ : (I) mixed hollow and simple drop formation, (II) single-core hollow drop formation and (III) multi-core hollow drop formation. The formation of pattern (I) is basically due to the insufficient flow rate of the inner gas jet whereas too high gas flow rates produce pattern (III). In patterns (I) and (III), drops are produced with non-uniformity in size because of irregularity in the formation of simple water drops (pattern (I)) and the number of gas cores enclosed in a large water shell (pattern (III)). In contrast, pattern (II) produces uniform-sized hollow drops that enclose only one gas core. The results obtained in pattern (II) further show that increasing $Q$ results in increasing the formation frequency and the hollow drop outer diameter, and in decreasing the shell wall thickness. Additionally, the measured breakup lengths in all patterns make plain that the outer jet breakup length varies complexly with $Q$ whereas the breakup length of the inner gas jet decreases with an increase in $Q$.

Moreover, we find a new feature observed in the formation and breakup of a hollow jet in pattern (II). This phenomenon relates to the merging of gas bubbles or the reunion of the inner gas jet with a just formed gas bubble within the outer jet of water. It is followed by the breakup of the outer jet into a single-core hollow drop. This formation affects considerably the formation frequency and outer diameter of the hollow drops. However, we have not studied it in detail in the present work. Therefore, in future work, we will focus on this phenomenon to find the ranges of $W e, R e$, and $Q$ at which it will occur, and how it is affected by the nozzle geometry as well as the hollow jet fluid properties.

\section{Acknowledgments}

This work was partially supported by the Japanese Government Ministry of Education, Culture, Sports, Science and Technology (MEXT) and Society for the Advanced Science and Technology at Ritsumeikan (ASTER). The authors would like to thank Prof. Shunji Homma at Saitama University and Dr. Thien D. Nguyen at Ritsumeikan University for 
helpful discussions.

\section{References}

(1) Kendall, J. M., Lee, M. C. and Wang, T. G., Metal Shell Technology based upon Hollow Jet Instability, Journal of Vacuum Science and Technology, Vol.20, No.4(1982), pp.1091-1093.

(2) Vu, T. V., Takakura, H., Wells, J. C. and Minemoto, T., Production of Hollow Spheres of Eutectic Tin-Lead Solder through a Coaxial Nozzle, Journal of Solid Mechanics and Materials Engineering, Vol.4, No.10(2010), pp.1530-1538.

(3) Heinzl, J. and Hertz, C., Ink-Jet Printing, Advances in Electronics and Electron Physics, Vol.65(1985), pp.91-171.

(4) Hertz, C. H. and Hermanrud, B., A Liquid Compound Jet, Journal of Fluid Mechanics, Vol.131(1983), pp.271-287.

(5) Denn, M. M., Continuous Drawing of Liquids to Form Fibers, Annual Review of Fluid Mechanics, Vol.12, No.1(1980), pp.365-387.

(6) Chang, F. and Su, Y., Controlled Double Emulsification Utilizing 3D PDMS Microchannels, Journal of Micromechanics and Microengineering, Vol.18, No.6(2008), pp.065018_1-8.

(7) Loscertales, I. G., Barrero, A., Guerrero, I., Cortijo, R., Marquez, M. and Gannan-Calvo, A. M., Micro/Nano Encapsulation via Electrified Coaxial Liquid Jets, Science, Vol.295, No.5560(2002), pp.1695-1698.

(8) López-Herrera, J. M., Barrero, A., López, A., Loscertales, I. G. and Márquez, M., Coaxial Jets Generated from Electrified Taylor Cones. Scaling Laws, Journal of Aerosol Science, Vol.34, No.5(2003), pp.535-552.

(9) Loscertales, I. G., Barrero, A., Márquez, M., Spretz, R., Velarde-Ortiz, R. and Larsen, G., Electrically Forced Coaxial Nanojets for One-Step Hollow Nanofiber Design, Journal of the American Chemical Society, Vol.126, No.17(2004), pp.5376-5377.

(10) Rayleigh, L., On the Capillary Phenomena of Jets, Proceedings of the Royal Society of London, Vol.29, No.196-199(1879), pp.71-97.

(11) Chauhan, A., Maldarelli, C., Rumschitzki, D. S. and Papageorgiou, D. T., Temporal and Spatial Instability of an Inviscid Compound Jet, Rheologica Acta, Vol.35, No.6(1996), pp.567-583.

(12) Shen, J. and Li, X., Instability of an Annular Viscous Liquid Jet, Acta Mechanica, Vol.114, No.1-4(1996), pp.167-183.

(13) Chen, J. N. and Lin, S. P., Instability of an Annular Jet Surrounded by a Viscous Gas in a Pipe, Journal of Fluid Mechanics, Vol.450(2002), pp.235-258.

(14) Zhou, C., Yue, P. and Feng, J. J., Formation of Simple and Compound Drops in Microfluidic Devices, Physics of Fluids, Vol.18, No.9(2006), pp.092105_1-14.

(15) Suryo, R., Doshi, P. and Basaran, O. A., Nonlinear Dynamics and Breakup of Compound Jets, Physics of Fluids, Vol.18, No.8(2006), pp.082107_1-31.

(16) Vu, T. V., Homma, S., Wells, J. C., Takakura, H. and Tryggvason, G., Numerical Simulation of Formation and Breakup of a Three-Fluid Compound Jet, Journal of Fluid Science and Technology, Vol.6, No.2, pp.252-263.

(17) Utada, A. S., Lorenceau, E., Link, D. R., Kaplan, P. D., Stone, H. A. and Weitz, D. A., Monodisperse Double Emulsions Generated from a Microcapillary Device, Science, Vol.308, No.5721(2005), pp.537 -541.

(18) Chiu, S. and Lin, T., Breakup of Compound Liquid Jets under Periodic Excitation at Small Core-to-Shell Mass Ratios, Journal of the Chinese Institute of Engineers, Vol.31, No.1(2008), pp.1-8.

(19) Kendall, J. M., Experiments on Annular Liquid Jet Instability and on the Formation of Liquid Shells, Physics of Fluids, Vol.29, No.7(1986), pp.2086-2094. 
(20) Li, X. and Shen, J., Experiments on Annular Liquid Jet Breakup, Atomization and Sprays, Vol.11, No.5(2001), pp.557-573.

(21) Yang, X., Feng, J. J., Liu, C. and Shen, J., Numerical Simulations of Jet Pinching-off and Drop Formation Using an Energetic Variational Phase-Field Method, Journal of Computational Physics, Vol.218, No.1(2006), pp.417-428.

(22) Richards, J. R., Lenhoff, A. M. and Beris, A. N., Dynamic Breakup of Liquid-Liquid Jets, Physics of Fluids, Vol.6, No.8(1994), pp.2640-2654.

(23) Herrada, M. A., Montanero, J. M., Ferrera, C. and Gannan-Calvo, A. M., Analysis of the Dripping to Jetting Transition in Compound Capillary Jets, Journal of Fluid Mechanics, Vol.649(2010), pp.523-536.

(24) Boulton-Stone, J. M. and Blake, J. R., Gas Bubbles Bursting at a Free Surface, Journal of Fluid Mechanics, Vol.254, No.1(2006), pp.437-466. 